\title{
Determining the mesh size of polymer solutions via the pore size distribution
}

\author{
Valerio Sorichetti, ${ }^{*},, \ddagger$ Virginie Hugouvieux,,$+\ddagger$ and Walter Kob*, ${ }^{*}, \uparrow$ \\ †Laboratoire Charles Coulomb (L2C), Univ. Montpellier, CNRS, F-34095, Montpellier, \\ France \\ $\ddagger$ IATE, INRAE, CIRAD, Montpellier SupAgro, Univ. Montpellier, F-34060, Montpellier, \\ France \\ IInstitut Universitaire de France \\ E-mail: valerio.sorichetti@umontpellier.fr; virginie.hugouvieux@inrae.fr; \\ walter.kob@umontpellier.fr
}




\section{Supporting Information}

\section{SI Mean-squared displacement}

In order to check that the time we used to equilibrate the system is sufficiently long, we consider two quantities: the mean-squared displacement (MSD) of the monomers, which we compute as ${ }^{1}$

$$
g_{1}(t) \equiv \frac{1}{(N / 2+1)} \sum_{i=N / 4}^{3 N / 4}\left\langle\left[\mathbf{r}_{i}(t)-\mathbf{r}_{i}(0)\right]^{2}\right\rangle,
$$

and the MSD of the centers of mass of the chains,

$$
g_{3}(t) \equiv\left\langle\left[\mathbf{r}_{\mathrm{cm}}(t)-\mathbf{r}_{\mathrm{cm}}(0)\right]^{2}\right\rangle,
$$

where $\mathbf{r}_{\mathrm{cm}}(t)$ is the position vector of the center of mass of the chain and $\langle\cdot\rangle$ denotes, as usual, the thermodynamic average. Note that in Eq. (S1), only the central half of the monomers belonging to a chain are considered, in order to suppress the fluctuations caused by chain ends. ${ }^{1}$

In the present work, the motion of every monomer is governed by a Langevin equation, and hydrodynamic interactions between monomers are neglected. Therefore, in the absence of entanglements, the Rouse model ${ }^{2,3}$ gives a good approximation of chain dynamics at all densities, whereas in the presence of entanglements, the dynamics is described by the reptation model. ${ }^{2,3}$ In both cases, when $t>t_{\text {rel }}$, with $t_{\text {rel }}$ the longest relaxation time of the system, we expect $g_{1}(t)=g_{3}(t) \propto t:^{1,3}$ Therefore, we can check that the system has equilibrated by verifying that this condition is met at long times.

In Fig. S1, we show $g_{1}(t)$ and $g_{3}(t)$ for different values of $N$ and $\rho$. For $N=50$ the 

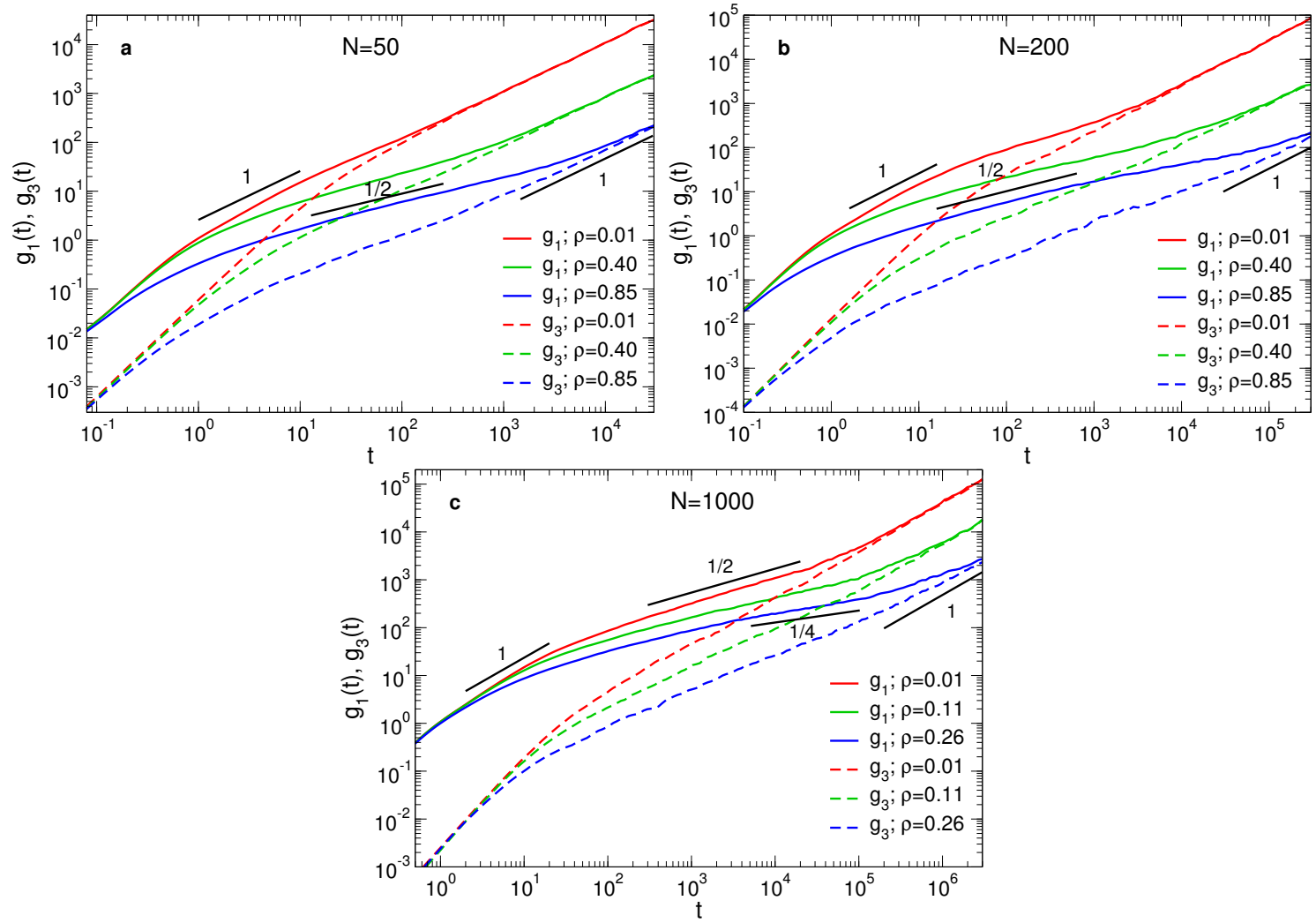

Figure S1: Mean squared displacement of the monomers, $g_{1}$ (Eq. (S1)), and of the centers of mass of the chains, $g_{3}$ (Eq. (S2)), for chain lengths $N=50$ (a), $N=200$ (b) and $N=1000$ (c) and different monomer densities $\rho$. Slopes are reported for comparison with the Rouse model and the reptation model. ${ }^{2,3}$ 
behavior of $g_{1}$ and $g_{3}$ is in qualitative agreement with the predictions of the Rouse model, and therefore the system is unentangled. For $N=200$ and 1000, $g_{1}$ and $g_{3}$ follow approximately the reptation model, ${ }^{2,3}$ thus the system is entangled. This is also evidenced by the comparison with the slopes reported in Fig. S1.

For all values of $N$ and $\rho$ considered, we observe that for large times, $g_{1}(t) \simeq g_{3}(t)$, and thus we conclude that the equilibration time $t_{e}$ we used (see Tab. 1 in the main text) is larger than the longest relaxation time of the system, $t_{\text {rel }}$, and therefore all the systems are well equilibrated.

\section{SII Radius of gyration and bond-bond correlation func- tion}

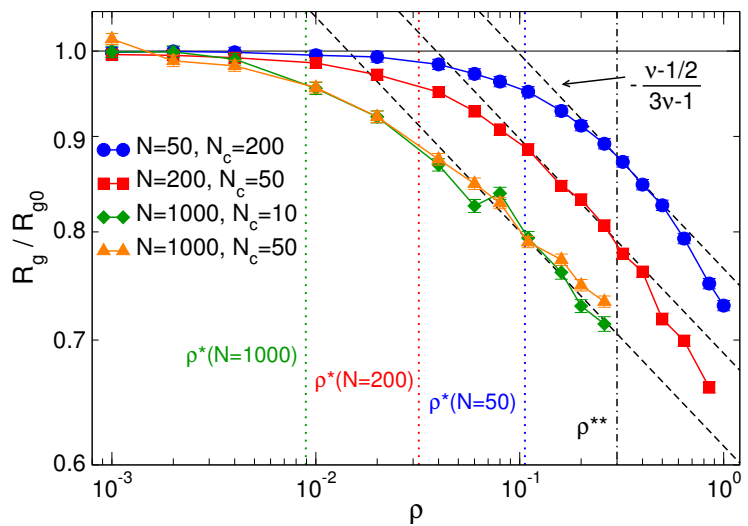

Figure S2: Normalized polymer radius of gyration, Eq. (S3), as a function of monomer density $\rho$. The dashed lines have slope $-\frac{\nu-1 / 2}{3 \nu-1} \simeq-0.115(\nu \simeq 0.588)$. The overlap densities have been estimated as $\rho^{*}(N)=3 N /\left(4 \pi R_{g 0}^{3}\right)$.

As a preliminary analysis of the structural properties of the simulated systems, we have studied the behavior of the radius of gyration $R_{g}$ as a function of monomer density $\rho$. The radius of gyration is defined as 


$$
R_{g}^{2} \equiv \frac{1}{N} \sum_{i=1}^{N}\left\langle\left(\mathbf{r}_{i}-\mathbf{r}_{\mathrm{cm}}\right)^{2}\right\rangle
$$

where $\mathbf{r}_{\mathrm{cm}}$ is the position of the center of mass of the chain and $\langle\cdot\rangle$ represents the thermodynamic average. Scaling theory predicts the dependence of $R_{g}$ on $\rho$ to be $\mathrm{e}^{2,4,5}$

$$
\frac{R_{g}}{R_{g 0}} \approx \begin{cases}1 & \rho<\rho^{*} \\ \left(\rho / \rho^{*}\right)^{-\frac{\nu-1 / 2}{3 \nu-1}} & \rho^{*}<\rho<\rho^{* *} \\ N^{1 / 2-\nu} & \rho>\rho^{* *},\end{cases}
$$

where $R_{g 0} \equiv \lim _{\rho \rightarrow 0} R_{g}(\rho)$ and $\rho^{*}$ is the overlap density.

Table S1: $N$-dependent properties of the simulated systems: Radius of gyration in the dilute limit, $R_{g 0}$ and approximate overlap concentration, calculated as $\rho^{*}=3 N /\left(4 \pi R_{g 0}^{3}\right)$.

\begin{tabular}{ccc}
\hline$N$ & $R_{g 0}$ & $\rho^{*}$ \\
\hline 50 & $4.83 \pm 0.02$ & $1.1 \cdot 10^{-1}$ \\
200 & $11.44 \pm 0.07$ & $3.2 \cdot 10^{-2}$ \\
1000 & $29.9 \pm 0.2$ & $8.9 \cdot 10^{-3}$ \\
\hline
\end{tabular}

In Fig. S2, we report $R_{g} / R_{g 0}$ as a function of $\rho$, with the dashed lines representing power laws with exponent $-\frac{\nu-1 / 2}{3 \nu-1} \simeq-0.115$. The radius of gyration in the dilute limit, $R_{g 0}$, was computed by simulating a single polymer chain at very low density ( $\rho=10^{-3}$ for $N=50,200$ and $\rho=10^{-4}$ for $\left.N=1000\right)$ : The results are reported in Tab. S1, alongside with the resulting estimate for the overlap density, $\rho^{*}=3 N /\left(4 \pi R_{g 0}^{3}\right)$. Figure $\mathrm{S} 2$ shows that $R_{g} / R_{g 0}$ follows the scaling predictions for all values of $N$ up to the density $\rho \simeq 0.3=\rho^{* *}$. For $\rho \gtrsim \rho^{* *}$, the decrease is steeper than what is predicted by scaling theory. This is likely due to the fact that in this density range the effective bond length $b$ decreases with increasing density, an effect which scaling theory does not take into account since one usually assumes that $b$ 
is density-independent. The concentrated regime behavior in Eq. (S4) should therefore be replaced by the expression

$$
R_{g} \approx R_{g 0}\left[\frac{b(\rho)}{b_{0}}\right] N^{1 / 2-\nu}
$$

where $b(\rho)$ is a decreasing function of $\rho$ and $b_{0}$ is the effective bond length of an isolated chain. The $\rho$-dependence of $b$ can be understood by studying the bond-bond correlation function $\left\langle\cos \left(\theta_{s}\right)\right\rangle$, where $\cos \left(\theta_{s}\right)$ is defined as ${ }^{6}$

$$
\cos \left(\theta_{s}\right) \equiv \frac{\mathbf{b}_{n} \cdot \mathbf{b}_{n+s}}{\left|\mathbf{b}_{n}\right|\left|\mathbf{b}_{n+s}\right|} \quad(s=1, \ldots, N-n),
$$

where $\mathbf{b}_{n} \equiv \mathbf{r}_{n+1}-\mathbf{r}_{n}$ is the $n$-th bond vector, with $\mathbf{r}_{n}$ the monomer's position vector.
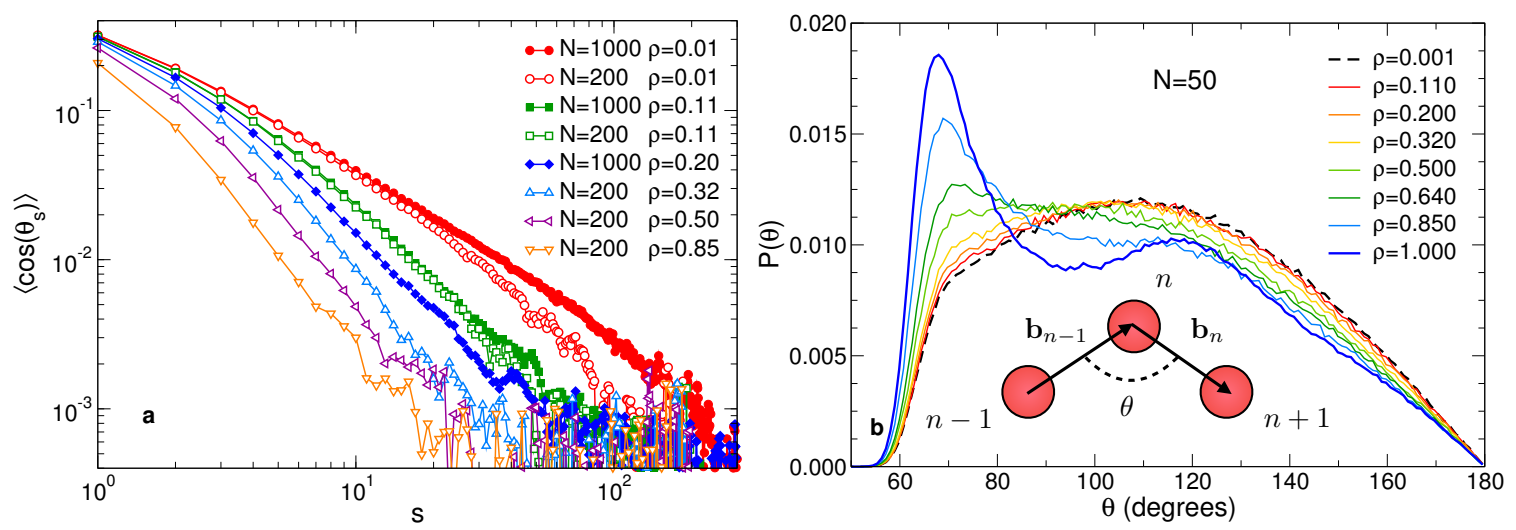

Figure S3: (a) Bond-bond correlation function $\left\langle\cos \left(\theta_{s}\right)\right\rangle$, Eq. (S6), for $N=200$ and 1000 and for different values of $\rho$. (b) Bond angle distribution for $N=50$ and different monomer densities $\rho$. We note that $\theta \equiv \pi-\theta_{1}$, see Eq. (S7). Cartoon: schematic representation of how $\theta$ is defined.

In Fig. S3a we report $\left\langle\cos \left(\theta_{s}\right)\right\rangle$ for $N=200$ and 1000 and different densities (for $N=50$ we find similar results). This data shows that with increasing $\rho$ the correlation function $\left\langle\cos \left(\theta_{s}\right)\right\rangle$ decreases faster, a behavior that is compatible with a reduction of the effective bond length $b$. Thus this observation explains qualitatively the high- $\rho$ behavior of $R_{g}$ observed in 
Fig. S2.

A reduction of $b$ with increasing density can also be inferred by considering the distribution $P(\theta)$ of the bond angle $\theta$, which we report in Fig. S3b for $N=50$ and different densities (for larger $N$, the results are basically the same).

The bond angle $\theta$ is defined as

$$
\theta \equiv \arccos \left(-\frac{\mathbf{b}_{n-1} \cdot \mathbf{b}_{n}}{\left|\mathbf{b}_{n-1}\right|\left|\mathbf{b}_{n}\right|}\right)=\pi-\theta_{1} \quad(n=1, \ldots, N)
$$

where $\theta_{1} \equiv \theta_{s=1}$, Eq. (S6). A schematic representation of how $\theta$ is defined is reported in Fig. S3b.

At low density, $P(\theta)$ shows a maximum at $\theta \simeq 112^{\circ}$ and falls to zero rather sharply at $\theta \simeq 60^{\circ}$ because of the excluded volume interaction. ${ }^{7}$ When $\rho$ is increased past $\rho \simeq 0.3$, the shape of $P(\theta)$ starts to change significantly in that it develops a peak at $\theta \simeq 70^{\circ}$, signaling that the chains are compressed. ${ }^{1,7}$ Overall, the average bond angle decreases by $\simeq 8 \%$ when going from the dilute regime to density $\rho \gtrsim 0.85$. This effect is also observable in the average bond length (not shown), although the decrease is in this case only $\simeq 1 \%$. Although it is possible to conceive chain conformations with a small $\langle\theta\rangle$ but a high $b$ (e.g., an "accordionlike" rigid polymer), the fact that up to very high $\rho$ the form factor is compatible with the scaling predictions for chains in a concentrated solution (Sec. SIV) suggests that the reduction of $\langle\theta\rangle$ corresponds to a reduction of the effective bond length $b$, as also observed from $\left\langle\cos \left(\theta_{s}\right)\right\rangle$.

\section{SIII Monomer structure factor}

In Fig. S4 we show the monomer structure factor for $N=50$ and $N=1000$ and for different densities, and the single chain structure factor $S_{1}(q)$ for $\rho=10^{-3}$ (thin continuous line, see also Sec. SIV). One finds that at this density $S(q) \simeq S_{1}(q)$ for $N=50$, i.e. the system is in 

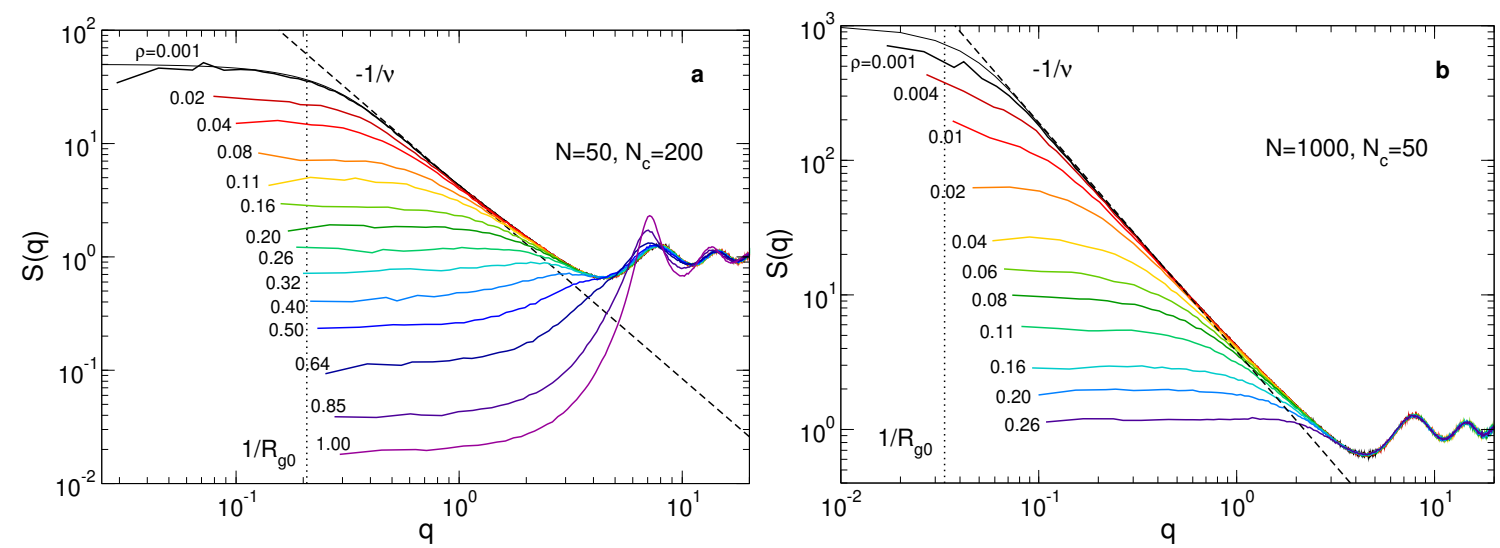

Figure S4: Monomer structure factor $S(q)$ for $N=50$ (a) and $N=1000$ (b), at different monomer densities. Thin continuous lines: $S_{1}(q)$ for $\rho=10^{-3}$. Dashed lines: slope $-1 / \nu \simeq$ $-1.70(\nu \simeq 0.588)$.

the dilute limit, whereas for $N=1000$ this limit is not yet reached. From our data at low and intermediate $\rho$, we can observe at intermediate $q$ a power law with slope $-1 / \nu \simeq-1.70$; as expected, this regime is more clearly observable for the longest chains, $N=1000$ (Fig. S4c).

As $\rho$ is increased, the isothermal compressibility $\kappa_{T}=S(0) / \rho k_{B} T$ decreases and eventually, for $\rho \geq 0.85$, the structure becomes virtually indistinguishable from that of a dense liquid. ${ }^{8,9}$

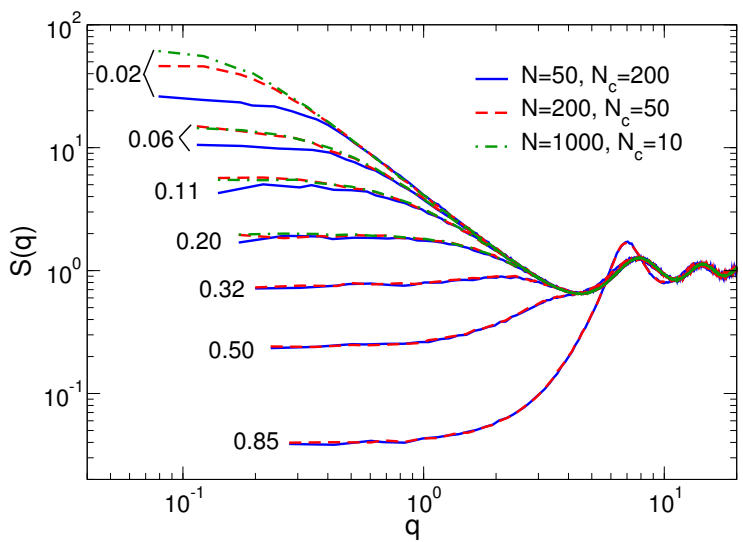

Figure S5: Comparison between the monomer structure factors $S(q)$ of systems with different chain lengths $N$ at the same monomer density $\rho$. For densities $\rho>0.11 \simeq \rho^{*}(N=50), S(q)$ becomes independent of $N$.

Comparing $S(q)$ for different chain lengths, we note that for densities $\rho>0.11 \simeq \rho^{*}(N=$ 50), $S(q)$ becomes independent of $N$. This is in demonstrated in Fig. S5, where we compare 
the monomer structure factor $S(q)$ for systems with the same density $\rho$ but with different chain lengths $N$. One can see that for densities $\rho \gtrsim 0.11$, which is approximately the overlap density of the $N=50$ system, $\rho^{*}(N=50) \simeq 0.106$ (see Tab. 1 in the main text), $S(q)$ is independent of $N$. This is expected, since for $\rho>\rho^{*}(N=50)$ all the systems here considered are in the semidilute regime, where the global structure is independent of chain length. ${ }^{2}$

\section{SIV Chain form factor}

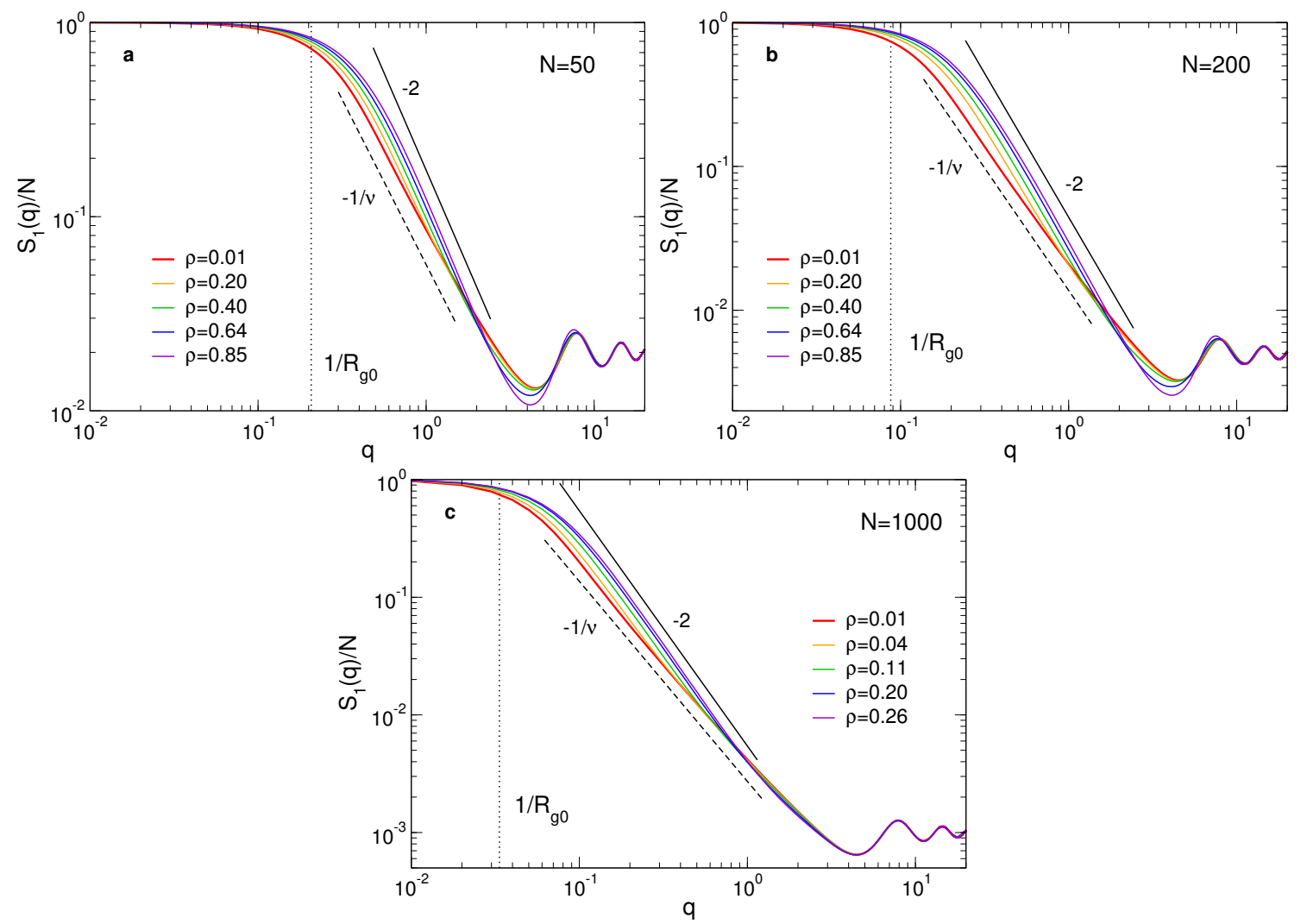

Figure S6: Single chain form factor for chain lengths $N=50$ (a), $N=200$ (b) and $N=1000$ (c) and different monomer densities $\rho$. Continuous lines: slope -2 (ideal chain). Dashed lines: slope $-1 / \nu \simeq-1.70$ (swollen chain).

In Fig. S6, we show the chain form factor $S_{1}(q) / N$ for $N=50,200$, and 1000 and for different values of the monomer density $\rho$. We recall that the theoretical prediction for $S_{1}(q) / N$ is ${ }^{2,5,10}$ 


$$
\frac{S_{1}(q)}{N}= \begin{cases}1 /\left(1+q^{2} R_{g}^{2} / 3\right) & q \ll R_{g}^{-1} \\ A q^{-2} & R_{g}^{-1} \ll q \ll \xi_{c}^{-1} \\ B q^{-1 / \nu} & \xi_{c}^{-1} \ll q \ll b^{-1} \\ \mathcal{O}(1 / N) & q \gg b^{-1},\end{cases}
$$

where $A, B>0$ are constants. In the dilute regime, $\xi_{c} \approx R_{g 0}$ and therefore the regime $S_{1}(q) / N \propto q^{-2}$ disappears; in the concentrated/melt regime, $\xi_{c} \approx b$ and therefore the regime $S_{1}(q) / N \propto q^{-1 / \nu}$ is not present (here $\nu=0.588$ is the Flory exponent for a good solvent).

Swollen chain behavior $S_{1}(q) / N \propto q^{-1 / \nu}$ and the ideal chain behavior $S_{1}(q) / N \propto q^{-2}$ are clearly observable in Fig. S6 at, respectively, low and high densities for $N=50$ and $N=200$. At intermediate densities, we expect the form factor to make a transition from a $q^{-2}$ dependence to a $q^{-1 / \nu}$ dependence at $q \approx 1 / \xi_{c} \cdot{ }^{2,4}$ Since $S_{1}(q) / N$ takes values from $\approx 1 / N$ to 1 , this transition is more easily observable for the longer chains: $N=200$ and $N=1000$.

\section{SV Numerical estimates of the mesh size}

In Tabs. S2-S4, we report for the simulated systems the numerical values of three different estimates of the mesh size: the correlation length (measured from the monomer radial distribution function $g(r)$, see Sec. 4.1 in the main text), $\xi_{c}$, the mean value of Torquato's PSD, $\langle r\rangle_{T}$ and that of Gubbins's PSD, $\langle r\rangle_{G}$. We also report the values of the monomer density $\rho$, of the monomer density normalized by the overlap density $\rho^{*}$ (see Tab. 2 in the main text) and of the scaling variable $\chi$, which, we recall, is operatively defined as $\chi=N\left(l_{b}^{3} \rho\right)^{1 /(3 \nu-1)}$, where $l_{b}$ is the root-mean squared (RMS) bond length measured from the configurations. The RMS bond length is basically independent on $\rho$ and has the value $l_{b} \simeq 0.97$ for the 
density range considered here. We recall that $N_{c}$ is the total number of chains in the system.

Table S2: Data for $N=50, N_{c}=200$. The overlap density for $N=50$ is $\rho^{*} \simeq 0.11$.

\begin{tabular}{cccccc}
\hline$\rho$ & $\rho / \rho^{*}$ & $\chi$ & $\xi_{c}$ & $\langle r\rangle_{T}$ & $\langle r\rangle_{G}$ \\
\hline 0.001 & 0.009 & 0.005 & 3.70 & 15.559 & 30.397 \\
0.002 & 0.018 & 0.013 & 3.61 & 11.498 & 22.798 \\
0.004 & 0.036 & 0.032 & 3.45 & 8.328 & 16.827 \\
0.010 & 0.091 & 0.107 & 3.16 & 5.120 & 10.668 \\
0.020 & 0.182 & 0.266 & 2.55 & 3.435 & 7.275 \\
0.040 & 0.364 & 0.660 & 1.84 & 2.182 & 4.712 \\
0.060 & 0.545 & 1.122 & 1.40 & 1.631 & 3.573 \\
0.080 & 0.727 & 1.634 & 1.06 & 1.314 & 2.904 \\
0.110 & 1.000 & 2.473 & 0.74 & 1.020 & 2.275 \\
0.160 & 1.455 & 4.050 & 0.64 & 0.750 & 1.688 \\
0.200 & 1.818 & 5.406 & 0.53 & 0.618 & 1.396 \\
0.260 & 2.364 & 7.626 & 0.42 & 0.486 & 1.107 \\
0.320 & 2.909 & 9.985 & 0.41 & 0.396 & 0.911 \\
0.400 & 3.636 & 13.352 & 0.42 & 0.315 & 0.730 \\
0.500 & 4.545 & 17.860 & 0.44 & 0.246 & 0.574 \\
0.640 & 5.818 & 24.562 & 0.71 & 0.181 & 0.428 \\
0.850 & 7.727 & 35.098 & 0.80 & 0.121 & 0.289 \\
1.000 & 9.091 & 42.754 & 1.16 & 0.092 & 0.222 \\
\hline
\end{tabular}


Table S3: Data for $N=200, N_{c}=50$. The overlap density for $N=200$ is $\rho^{*} \simeq 0.032$.

\begin{tabular}{cccccc}
\hline$\rho$ & $\rho / \rho^{*}$ & $\chi$ & $\xi_{c}$ & $\langle r\rangle_{T}$ & $\langle r\rangle_{G}$ \\
\hline 0.001 & 0.031 & 0.021 & 8.88 & 21.864 & 43.651 \\
0.002 & 0.063 & 0.052 & 8.53 & 15.402 & 31.524 \\
0.004 & 0.125 & 0.130 & 7.59 & 10.614 & 22.069 \\
0.010 & 0.313 & 0.430 & 5.72 & 6.009 & 12.985 \\
0.020 & 0.625 & 1.059 & 4.14 & 3.821 & 8.251 \\
0.040 & 1.250 & 2.640 & 2.48 & 2.315 & 5.060 \\
0.060 & 1.875 & 4.492 & 1.81 & 1.709 & 3.740 \\
0.080 & 2.500 & 6.537 & 1.36 & 1.351 & 3.006 \\
0.110 & 3.438 & 9.928 & 0.85 & 1.046 & 2.336 \\
0.160 & 5.000 & 16.210 & 0.62 & 0.762 & 1.716 \\
0.200 & 6.250 & 21.615 & 0.54 & 0.622 & 1.414 \\
0.260 & 8.125 & 30.518 & 0.46 & 0.488 & 1.118 \\
0.320 & 10.000 & 40.114 & 0.43 & 0.400 & 0.917 \\
0.400 & 12.500 & 53.366 & 0.47 & 0.316 & 0.733 \\
0.500 & 15.625 & 71.204 & 0.52 & 0.246 & 0.576 \\
0.640 & 20.000 & 98.540 & 0.63 & 0.181 & 0.429 \\
0.850 & 26.563 & 140.077 & 0.82 & 0.121 & 0.289 \\
\hline
\end{tabular}


Table S4: Data for $N=1000$. The overlap density for $N=1000$ is $\rho^{*} \simeq 0.0089$.

(a) $N_{c}=10$.

\begin{tabular}{cccccc}
\hline$\rho$ & $\rho / \rho^{*}$ & $\chi$ & $\xi_{c}$ & $\langle r\rangle_{T}$ & $\langle r\rangle_{G}$ \\
\hline 0.001 & 0.112 & 0.106 & 16.98 & 28.626 & 59.549 \\
0.002 & 0.225 & 0.262 & 14.84 & 19.343 & 40.196 \\
0.004 & 0.449 & 0.649 & 12.24 & 12.349 & 26.436 \\
0.010 & 1.124 & 2.145 & 8.10 & 6.589 & 14.252 \\
0.020 & 2.247 & 5.302 & 4.96 & 3.972 & 8.658 \\
0.040 & 4.494 & 13.165 & 2.75 & 2.350 & 5.164 \\
0.060 & 6.742 & 22.398 & 1.83 & 1.720 & 3.793 \\
0.080 & 8.989 & 32.628 & 1.40 & 1.365 & 3.035 \\
0.110 & 12.360 & 49.473 & 0.85 & 1.052 & 2.348 \\
0.160 & 17.978 & 81.051 & 0.77 & 0.764 & 1.723 \\
0.200 & 22.472 & 108.430 & 0.53 & 0.625 & 1.419 \\
0.260 & 29.213 & 152.821 & 0.45 & 0.490 & 1.120 \\
\hline
\end{tabular}

(b) $N_{c}=50$.

\begin{tabular}{cccccc}
\hline$\rho$ & $\rho / \rho^{*}$ & $\chi$ & $\xi_{c}$ & $\langle r\rangle_{T}$ & $\langle r\rangle_{G}$ \\
\hline 0.001 & 0.112 & 0.105 & 17.86 & 28.851 & 59.726 \\
0.002 & 0.225 & 0.261 & 15.15 & 19.837 & 42.160 \\
0.004 & 0.449 & 0.648 & 12.50 & 12.599 & 26.772 \\
0.010 & 1.124 & 2.153 & 8.59 & 6.650 & 14.390 \\
0.020 & 2.247 & 5.325 & 4.93 & 3.991 & 8.663 \\
0.040 & 4.494 & 13.192 & 2.83 & 2.357 & 5.178 \\
0.060 & 6.742 & 22.396 & 1.83 & 1.718 & 3.793 \\
0.080 & 8.989 & 32.626 & 1.33 & 1.365 & 3.033 \\
0.110 & 12.360 & 49.620 & 0.85 & 1.050 & 2.347 \\
0.160 & 17.978 & 80.910 & 0.79 & 0.765 & 1.722 \\
0.200 & 22.472 & 108.311 & 0.56 & 0.625 & 1.419 \\
0.260 & 29.213 & 152.587 & 0.47 & 0.490 & 1.120 \\
\hline
\end{tabular}




\section{References}

(1) Hsu, H.-P.; Kremer, K. Static and dynamic properties of large polymer melts in equilibrium. J. Chem. Phys. 2016, 144, 154907.

(2) Rubinstein, M.; Colby, R. H. Polymer physics; Oxford University Press, 2003.

(3) Doi, M.; Edwards, S. F. The theory of polymer dynamics; Oxford University Press, 1988.

(4) De Gennes, P.-G. Scaling concepts in polymer physics; Cornell University Press, 1979.

(5) Teraoka, I. Polymer solutions: an introduction to physical properties; John Wiley \& Sons, 2002.

(6) Wittmer, J.; Meyer, H.; Baschnagel, J.; Johner, A.; Obukhov, S.; Mattioni, L.; Müller, M.; Semenov, A. N. Long range bond-bond correlations in dense polymer solutions. Physical review letters 2004, 93, 147801.

(7) Schnell, B.; Meyer, H.; Fond, C.; Wittmer, J.; Baschnagel, J. Simulated glass-forming polymer melts: Glass transition temperature and elastic constants of the glassy state. The European Physical Journal E 2011, 34, 97.

(8) Hansen, J.-P.; McDonald, I. R. Theory of simple liquids; Elsevier, 1990.

(9) Verlet, L. Computer "experiments" on classical fluids II - Equilibrium correlation functions. Phys. Rev. 1968, 165, 201.

(10) Paul, W.; Binder, K.; Heermann, D. W.; Kremer, K. Crossover scaling in semidilute polymer solutions: a Monte Carlo test. J. Phys. II 1991, 1, 37-60. 